\title{
A FOURIER-NEUMANN SERIES AND ITS APPLICATION TO THE REDUCTION OF TRIPLE COSINE SERIES
}

\author{
by C. J. TRANTER and J. C. COOKE
}

(Received 1 May, 1972)

1. Introduction. The Jacobi expansion

$$
\sin \left(z \sin \frac{1}{2} x\right)=2 \sum_{n=0}^{\infty} J_{2 n+1}(z) \sin \left(n+\frac{1}{2}\right) x
$$

is well known and easily obtained from the generating function of the Bessel coefficients. The sum of the series on the right of equation (1) when $\sin \left(n+\frac{1}{2}\right) x$ is replaced by $\cos \left(n+\frac{1}{2}\right) x$ cannot be found in this way but it can be expressed in terms of a definite integral as shown below. The result so obtained is useful in reducing certain triple cosine series to dual series and so simplifying the solution given by one of us for such series in an earlier paper [1].

2. The series $\sum_{n=0}^{\infty} J_{2 n+1}(z) \cos \left(n+\frac{1}{2}\right) x$. Starting with the result [2, p. 53]

$$
\sqrt{2} \sum_{n=0}^{\infty} \cos \left(n+\frac{1}{2}\right) x P_{n}(\cos u)= \begin{cases}(\cos x-\cos u)^{-1 / 2} & (0<x<u<\pi), \\ 0 & (0<u<x<\pi),\end{cases}
$$

and using the orthogonality relations for the Legendre polynomials, we have

Also [3, p. 140]

$$
\int_{x}^{\pi} \frac{\sin u P_{n}(\cos u) d u}{(\cos x-\cos u)^{1 / 2}}=\frac{\sqrt{2} \cos \left(n+\frac{1}{2}\right) x}{n+\frac{1}{2}} .
$$

$$
\frac{1}{2} z J_{0}\left(z \sin \frac{1}{2} u\right)=\sum_{n=0}^{\infty}(2 n+1) P_{n}(\cos u) J_{2 n+1}(z)
$$

when the hypergeometric function in Watson's formula is expressed as a Legendre polynomial. Multiplication of (3) by $\sin u(\cos x-\cos u)^{-1 / 2}$, integration with respect to $u$ between $x$ and $\pi$ and use of (2) then gives

$$
\sum_{n=0}^{\infty} J_{2 n+1}(z) \cos \left(n+\frac{1}{2}\right) x=\frac{z}{4 \sqrt{2}} \int_{x}^{\pi} \frac{\sin u J_{0}\left(z \sin \frac{1}{2} u\right) d u}{(\cos x-\cos u)^{1 / 2}}
$$

and this is the result required.

It is interesting to note that similar analysis gives

$$
\sum_{n=0}^{\infty} J_{2 n+1}(z) \sin \left(n+\frac{1}{2}\right) x=\frac{z}{4 \sqrt{ } 2} \int_{0}^{x} \frac{\sin u J_{0}\left(z \sin \frac{1}{2} u\right) d u}{(\cos u-\cos x)^{1 / 2}}
$$

and that, in this case, the integral on the right can be transformed into Sonine's first finite integral, which can then be evaluated $[3$, p. 373] to give the result (1). 
3. The reduction of triple cosine series. The coefficients $B_{n}$ are required to be found from the triple series

$$
\left.\begin{array}{ll}
\sum_{n=0}^{\infty} B_{n} \cos \left(n+\frac{1}{2}\right) x=0 & (0<x<\alpha \text { and } \beta<x<\pi), \\
\sum_{n=0}^{\infty}\left(n+\frac{1}{2}\right) B_{n} \cos \left(n+\frac{1}{2}\right) x=f(x) & (\alpha<x<\beta),
\end{array}\right\}
$$

where $f(x)$ is a prescribed function of $x$. We write

$$
B_{n}=\sum_{r=0}^{\infty} C_{r} \int_{0}^{\infty} J_{2 r+1}\left(z \sin \frac{1}{2} \beta\right) J_{2 n+1}(z) \frac{d z}{z}
$$

and reduce the problem to that of finding the coefficients $C_{r}$ in certain dual series.

Substituting for $B_{n}$ from (7), interchanging the order of summation and integration and using (4), we have

$$
\sum_{n=0}^{\infty} B_{n} \cos \left(n+\frac{1}{2}\right) x=\frac{1}{4 \sqrt{ } 2} \sum_{r=0}^{\infty} C_{r} \int_{x}^{\pi} \frac{\sin u d u}{(\cos x-\cos u)^{1 / 2}} \int_{0}^{\infty} J_{2 r+1}\left(z \sin \frac{1}{2} \beta\right) J_{0}\left(z \sin \frac{1}{2} u\right) d z .
$$

Now $[3$, p. 401]

$$
\int_{0}^{\infty} J_{2 r+1}\left(z \sin \frac{1}{2} \beta\right) J_{0}\left(z \sin \frac{1}{2} u\right) d z= \begin{cases}0 & (u>\beta), \\ \operatorname{cosec} \frac{1}{2} \beta_{2} F_{1}\left(r+1,-r ; 1 ; \sin ^{2} \frac{1}{2} u \operatorname{cosec}^{2} \frac{1}{2} \beta\right) & (u<\beta),\end{cases}
$$

so that (8) shows that

$$
\sum_{n=0}^{\infty} B_{n} \cos \left(n+\frac{1}{2}\right) x=0, \quad(x>\beta)
$$

and that, when $0<x<\beta$,

$$
\sum_{n=0}^{\infty} B_{n} \cos \left(n+\frac{1}{2}\right) x=\frac{\operatorname{cosec} \frac{1}{2} \beta}{4 \sqrt{2}} \sum_{r=0}^{\infty} C_{r} \int_{x}^{\beta} \frac{\sin u_{2} F_{1}\left(r+1,-r ; 1 ; \sin ^{2} \frac{1}{2} u \operatorname{cosec}^{2} \frac{1}{2} \beta\right) d u}{(\cos x-\cos u)^{1 / 2}} .
$$

The form chosen for $B_{n}$ in equation (7) is therefore such that the equation (5) when $\beta<x<\pi$ is automatically satisfied. The integral on the right of $(10)$ can be transformed by writing

$$
\sin \frac{1}{2} \theta=\sin \frac{1}{2} u \operatorname{cosec} \frac{1}{2} \beta, \quad \sin \frac{1}{2} y=\sin \frac{1}{2} x \operatorname{cosec} \frac{1}{2} \beta
$$

and expressing the hypergeometric function as a Legendre polynomial to give, when $0<x<\beta$,

$$
\begin{aligned}
\sum_{n=0}^{\infty} B_{n} \cos \left(n+\frac{1}{2}\right) x & =\frac{1}{4 \sqrt{2}} \sum_{r=0}^{\infty} C_{r} \int_{y}^{\pi} \frac{\sin \theta P_{r}(\cos \theta) d \theta}{(\cos y-\cos \theta)^{1 / 2}} \\
& =\frac{1}{4} \sum_{r=0}^{\infty} C_{r} \frac{\cos \left(r+\frac{1}{2} y\right)}{r+\frac{1}{2}} \quad(0<y<\pi),
\end{aligned}
$$

the last step resulting from use of equation (2).

G2 
Equation (6) can be expressed in terms of $C_{r}$ and $y$ as follows. Substituting from (7) in (6) and interchanging the orders of integration and summation, we have

$$
\sum_{r=0}^{\infty} C_{r} \int_{0}^{\infty} J_{2 r+1}\left(z \sin \frac{1}{2} \beta\right) \sum_{n=0}^{\infty}\left(n+\frac{1}{2}\right) J_{2 n+1}(z) \cos \left(n+\frac{1}{2}\right) x \frac{d z}{z}=f(x) .
$$

Differentiation of equation (1) with respect to $x$ gives

$$
\frac{1}{2} z \cos \frac{1}{2} x \cos \left(z \sin \frac{1}{2} x\right)=2 \sum_{n=0}^{\infty}\left(n+\frac{1}{2}\right) J_{2 n+1}(z) \cos \left(n+\frac{1}{2}\right) x,
$$

so that equation (13) becomes

$$
\frac{1}{4} \cos \frac{1}{2} x \sum_{r=0}^{\infty} C_{r} \int_{0}^{\infty} J_{2 r+1}\left(z \sin \frac{1}{2} \beta\right) \cos \left(z \sin \frac{1}{2} x\right) d z=f(x) \quad(\alpha<x<\beta) .
$$

Now [3, p. 405]

$$
\begin{aligned}
\int_{0}^{\infty} J_{2 r+1}\left(z \sin \frac{1}{2} \beta\right) \cos \left(z \sin \frac{1}{2} x\right) d z & =\frac{\cos \left\{(2 r+1) \sin ^{-1}\left(\sin \frac{1}{2} x \operatorname{cosec} \frac{1}{2} \beta\right)\right\}}{\left(\sin ^{2} \frac{1}{2} \beta-\sin ^{2} \frac{1}{2} x\right)^{1 / 2}} \\
& =\frac{\cos \left(r+\frac{1}{2}\right) y}{\sin \frac{1}{2} \beta \cos \frac{1}{2} y},
\end{aligned}
$$

when use is made of the second of equations (11). Hence, finally, equation (14) gives

$$
\sum_{r=0}^{\infty} C_{r} \cos \left(r+\frac{1}{2}\right) y=\frac{4 \sin \frac{1}{2} \beta \cos \frac{1}{2} y}{\left(1-\sin ^{2} \frac{1}{2} \beta \sin ^{2} \frac{1}{2} y\right)^{1 / 2}} f\left\{2 \sin ^{-1}\left(\sin \frac{1}{2} \beta \sin \frac{1}{2} y\right)\right\}
$$

and, as equation (6) holds when $\alpha<x<\beta$, equation (15) is valid when $\gamma<y<\pi$, where

$$
\sin \frac{1}{2} \gamma=\sin \frac{1}{2} \alpha \operatorname{cosec} \frac{1}{2} \beta \text {. }
$$

We have thus shown that the coefficients $B_{n}$ in the triple series equations (5) and (6) can be found through the relation (7) from the coefficients in the dual series

$$
\left.\begin{array}{ll}
\sum_{r=0}^{\infty} C_{r}\left(r+\frac{1}{2}\right)^{-1} \cos \left(r+\frac{1}{2}\right) y=0 & (0<y<\gamma), \\
\sum_{r=0}^{\infty} C_{r} \cos \left(r+\frac{1}{2}\right) y=\frac{4 \sin \frac{1}{2} \beta \cos \frac{1}{2} y}{\left(1-\sin ^{2} \frac{1}{2} \beta \sin ^{2} \frac{1}{2} y\right)^{1 / 2}} f\left\{2 \sin ^{-1}\left(\sin \frac{1}{2} \beta \sin \frac{1}{2} y\right)\right\} & (\gamma<y<\pi),
\end{array}\right\}
$$

the value of $\gamma$ being given by equation (16). It is perhaps worth noting that this method of reducing triple to dual series can also (by setting $\alpha=0$ ) reduce dual series to a single series over the range $(0, \pi)$ and that the coefficients in the latter series can be found by the usual Fourier analysis. 


\section{REFERENCES}

1. C. J. Tranter, Some triple trigonometrical series, Glasgow Math. J. 10 (1969), 121-125.

2. W. Magnus and F. Oberhettinger (translated by J. Wermer), Special functions of mathematical physics (New York, 1949).

3. G. N. Watson, Theory of Bessel functions (Cambridge, 1944).

Royal Military College of Science

UNIVERSITY OF BRISTOL

SHRIVENHAM 